OPEN ACCESS

Edited by:

Mark Billinghurst,

University of South Australia, Australia

Reviewed by:

Page Lyn Anderson,

Georgia State University,

United States

Hunter Hoffman,

University of Washington,

United States

*Correspondence:

Merve Dilgul

m.dilgul@qmul.ac.uk

${ }^{\dagger}$ Present Address: Merve Dilgul,

Lauren M. Hickling,

Stefan Priebe,

Victoria J. Bird,

Unit for Social and Community

Psychiatry, Newham Center for Mental

Health, London, United Kingdom;

Daniela Antonie,

Vicarage Lane Health Center, London, United Kingdom

Specialty section:

This article was submitted to Virtual Reality in Medicine, a section of the journal

Frontiers in Virtual Reality

Received: 23 September 2020

Accepted: 14 December 2020

Published: 25 January 2021

Citation:

Dilgul M, Hickling LM, Antonie $D$, Priebe $S$ and Bird VJ (2021) Virtual

Reality Group Therapy for the Treatment of Depression: A Qualitative

Study on Stakeholder Perspectives.

Front. Virtual Real. 1:609545.

doi: 10.3389/frvir.2020.609545

\section{Virtual Reality Group Therapy for the Treatment of Depression: A Qualitative Study on Stakeholder Perspectives}

\author{
Merve Dilgul ${ }^{1,2 * t}$, Lauren M. Hickling ${ }^{1,2+}$, Daniela Antonie ${ }^{3 \dagger}$, Stefan Priebe ${ }^{1,2 t}$ and \\ Victoria J. Bird ${ }^{1,2 t}$ \\ ${ }^{1}$ Unit for Social and Community Psychiatry, Institute for Population Health Sciences, Queen Mary University of London, London, \\ United Kingdom, ${ }^{2}$ East London NHS Foundation Trust, London, United Kingdom, ${ }^{3}$ Newham Talking Therapies, London, United Kingdom
}

Background: Cognitive behavioral group therapy alleviates depression by teaching patients to think and behave in more positive ways. Teletherapy (e.g., Zoom) is becoming more widely used, especially during the COVID-19 pandemic (where meeting in person is not safe). The current study explores the acceptability of taking teletherapy to the next level: Virtual Reality Group Therapy (VRGT).

Methods: Semistructured interviews were conducted to explore stakeholder views on VRGT. Ten depressed patients and ten therapists watched a demonstration video of the proposed VRGT intervention and tested the VR application using a stand-alone VR headset. In VRGT, patients will use an avatar to interact with each other and with their therapist via networked multiparticipant VR.

Results: Therapists and patients generally responded favorably to the idea of doing group therapy sessions in VR. Patients especially liked the idea of remaining anonymous via an avatar. Patients and therapists both indicated that the anonymity provided by avatars could increase patient's willingness to make disclosures (to talk more freely and honestly), which could increase participation and could lead to better group cohesion.

Conclusion: Although the findings suggested that VRGT may be more acceptable for some patients than for others, overall, the response of the patients and therapists was largely positive. Recommendations from this study could be used during the COVID-19 pandemic to deliver VRGTs. Finally, design ideas for creating a group VR world customdesigned for group therapy are discussed.

Keywords: virtual reality, digital intervention, depression, qualitative, treatment

\section{INTRODUCTION}

Group therapies are pragmatic as they allow the therapist to deliver therapy to multiple patients in a time-efficient manner (Wykes et al., 1999). Therapeutic benefits of the group format include group cohesiveness, imitative behavior, interpersonal learning, and recognition of similarities in others (Yalom and Crouch, 1990). This format of treatment is effective and is routinely recommended for the treatment of depression (McDermut et al., 2001; Kösters et al., 2006; Cuijpers et al., 2008). However, up to $40 \%$ of patients with depression do not attend or drop out of group treatment (Hans and Hiller, 2013). Reasons for poor attendance include group treatments not being accessible (e.g., 
time and location) and patients feeling socially anxious in a group (Dilgul et al., 2018). The rate and reasons for nonattendance indicate that there needs to be a variety of delivery methods to make therapy accessible to all patients that may benefit from it (Fernandez, Salem, Swift, and Ramtahal, 2015).

To improve access to psychological therapies, the United Kingdom's National Health Service (NHS) "Five Year Forward View of Mental Health" report has recommended the use of digitally enabled therapies (e.g., mobile application-based selfhelp) (NHS Mental Health Taskforce, 2016). Following the recommendation, digitally-enabled therapies are now increasingly being used in mental health care (Ralston et al., 2019). These therapies aim to reduce: (1) cost of services, (2) time and travel associated with treatment, and (3) the perceived stigma of having to go to a treatment center (Price et al., 2014; Fairburn and Patel, 2017; Bucci et al., 2019; Ralston et al., 2019). In reducing these potential barriers, these digitally enabled therapies aim to enhance access to evidence-based care and more actively engage patients in their treatment (Price et al., 2014).

Virtual reality (VR) is "a computer-generated digital environment that can be experienced and interacted with as if that environment was real" (Jerald, 2015). Users can access this computer-generated environment by inserting their smartphone into a head-mounted display (HMD), e.g., Samsung Gear VR. This technology is a feasible, acceptable, and cost-effective tool in the treatment of several different mental health disorders, with exposure-based treatments for patients with anxiety disorders having the largest evidence base (Freeman et al., 2017; MaplesKeller et al., 2017).

In 2013, psychotherapists had predicted that, by 2022, interventions using VR would be one of the most likely psychotherapy interventions to increase in popularity (Norcross et al., 2013). Since then, to mitigate the consequences of the coronavirus pandemic lockdowns, therapists have significantly increased their use of digitally enabled therapies to deliver treatment to their patients. This increase in the use of digitally enabled therapies and the investments in VR has made researchers predict that, soon, therapists and patients in physically different locations would use VR to "meet" in a shared computer-generated world designed for individual and group therapy sessions (Sampaio et al., 2020).

Despite encouraging predictions and rapid uptake of digitally enabled therapies, there is limited research into VR treatments for depression (Freeman et al., 2017; Dilgul et al., 2020). Furthermore, most VR research into mental health treatment has generally focused on its use one-to-one, in a clinic, with a health care professional (Dilgul et al., 2020). It is suggested that delivering VR treatments in this format is unlikely to improve treatment accessibility (Price et al., 2014; Fairburn and Patel, 2017; Bucci et al., 2019; Ralston et al., 2019).

VR has the potential to connect people in a virtual environment and could be used to deliver verbal group therapies (Sampaio et al., 2020). However, there is a lack of research on the use of VR in group treatments (Watson et al., 2008). Only two studies have used VR to deliver virtual groups remotely. Nosek et al. (2016) delivered a VR self-esteem enhancement psychoeducational intervention for women with physical disabilities, reporting improvements in patients' selfesteem and depressive symptomatology. Similarly, Knowles et al. (2017) delivered a VR grief support group for widowers and found that it significantly reduced patients' levels of depression. Despite their positive results on depression outcomes, these interventions were neither developed for patients with depression nor did they deliver cognitive behavioral group therapy (CBGT).

CBGT is a widely researched and empirically supported psychotherapeutic method (Fenn and Byrne, 2013). Metaanalyses have demonstrated the efficacy of CBGT, and studies have reported its cost-effectiveness in the treatment of depression (McDermut et al., 2001; Tucker and Oei, 2007; Huntley et al., 2012). As a result of this, in the United Kingdom, CBGT is recommended for the treatment and management of depression (NICE, 2018). Acceptability is the extent to which people delivering or receiving the healthcare intervention consider it to be appropriate for themselves (Sekhon, Cartwright, and Francis, 2017). Consequently, the acceptability of delivering or receiving CBGT, remotely using immersive VR technology for patients with depression, is unknown.

This qualitative study aims to assess the acceptability of a proposed VR group therapy (VRGT) intervention based on CBT.

This study will address two research questions:

(1) What are the views of potential stakeholders on cognitive behavioral group therapy delivered via virtual reality?

(2) How can a cognitive behavioral group therapy intervention be adapted to be delivered via virtual reality?

\section{METHODS}

\section{Research Approach}

Pragmatism is a philosophical worldview that is concerned with identifying what practically matters for a group of people (Williams, 2016). As this study was interested in exploring patients and therapists' views of a VR group intervention, including its acceptability in practice, the study was developed, conducted, and analyzed using a pragmatic approach. Semistructured interviews were conducted to explore the stakeholders' views (Yeo et al., 2014). This method allowed the researcher to use a topic guide to explore potential areas of interest and simultaneously created an opportunity for interviewees to discuss matters they considered appropriate (Arthur et al., 2014). Thematic analysis was used to identify themes in the data. Thematic analysis is a theoretically flexible approach to analyzing qualitative data (Braun and Clarke, 2006). The conduct and reporting of the study adhere to the Standards for Reporting Qualitative Research guidelines (O'Brien et al., 2014). The study received favorable ethics opinion from East Midlands-Leicester South committee (ref: 19/EM/0217).

\section{Researcher Characteristics}

This study was developed, conducted, and analyzed by a professionally diverse team. The credentials of all authors, 
TABLE 1 | Researcher characteristics and reflexivity.

\begin{tabular}{|c|c|c|c|c|c|}
\hline & Author 1 MD & Author 2 LH & Author 3 DA & Author 4 SP & Author 5 VB \\
\hline Professional role & Ph.D. student & $\begin{array}{l}\text { Live-experience } \\
\text { researcher }\end{array}$ & $\begin{array}{l}\text { Consultant clinical } \\
\text { psychologist }\end{array}$ & Professor, psychiatrist & $\begin{array}{l}\text { Professor, health } \\
\text { services researcher }\end{array}$ \\
\hline Role in research team & $\begin{array}{l}\text { Interviewer and lead } \\
\text { analyst }\end{array}$ & Second data analyst & Therapist recruitment & Ph.D. supervisor & Ph.D. supervisor \\
\hline \multirow[t]{3}{*}{$\begin{array}{l}\text { Possible influence on } \\
\text { interview conduct or } \\
\text { analysis }\end{array}$} & $\begin{array}{l}\text { Developing a VR group } \\
\text { therapy intervention (Ph.D.) }\end{array}$ & $\begin{array}{l}\text { Experience of attending } \\
\text { and facilitating group } \\
\text { therapy }\end{array}$ & $\begin{array}{l}\text { Knowledge and experience } \\
\text { of facilitating group therapies }\end{array}$ & $\begin{array}{l}\text { Knowledge of } \\
\text { intervention } \\
\text { development }\end{array}$ & $\begin{array}{l}\text { Familiarity with VR } \\
\text { literature }\end{array}$ \\
\hline & $\begin{array}{l}\text { Familiarity with VR mental } \\
\text { health literature }\end{array}$ & & & $\begin{array}{l}\text { Previous experience of } \\
\text { practicing as a clinician }\end{array}$ & $\begin{array}{l}\text { Knowledge of } \\
\text { intervention } \\
\text { development }\end{array}$ \\
\hline & $\begin{array}{l}\text { Experience of attending } \\
\text { and facilitating group } \\
\text { therapy }\end{array}$ & & & & \\
\hline
\end{tabular}

their roles within the research team, and their possible influence on the conduct or analysis of the study are presented in Table 1.

\section{Participants}

Participants in this study consisted of patients and therapists. Purposive sampling and snowballing were used to identify the participants. Patients were recruited using a poster advert on Twitter, and a phone call was made to patients from a list who had expressed interest in future research in a previous study of depression (Carr et al., 2017). Therapists were recruited through professional contacts of the authors (MD and $\mathrm{DA}$ ) and word of mouth from the therapists that were interviewed.

To be eligible to take part in the interviews, all participants had to be over 18 years old and able to communicate verbally in English. Patients were invited to participate if they had a diagnosis of depression in the last 12 months (ICD10: F32-39) and had the capacity to consent to be interviewed. This was assessed before the interviews using the 'assessing capacity to consent to participation in research' assessment tool developed by the British Psychological Society (Dobson, 2008). Patient eligibility was assessed via electronic patient records. If patients did not have an electronic patient record, eligibility was based on self-report via telephone. Patients were not eligible to participate if their diagnosis of depression included psychotic symptoms (ICD-10: F32.3 and F33.3), as there is no evidence yet on the suitability of remote virtual reality treatment for this population. Furthermore, patients were excluded if they had an organic or neurodevelopmental disorder or they were admitted to hospital during the study period.

In the United Kingdom, Improving Access to Psychological Therapies (IAPT) is a nationwide talking therapy program, which delivers the National Institute for Health and Care Excellence(NICE-) recommended psychological therapies (Clark, 2011; NHS, 2018). Therapists were invited to participate in the interviews if they were trained in the provision of low- or high-intensity IAPT interventions and if they had experience providing group $\mathrm{CBT}$ within community mental health settings.

To conduct a thematic analysis, Braun and Clarke (2013) recommend conducting six to ten interviews per study sample. As this study consisted of two samples, patient and therapist, recruitment continued until ten patients, and ten therapists were interviewed.

\section{The Proposed VRGT Intervention}

In this proposed intervention, patients with depression and the therapist would access the CBGT remotely from home using an HMD, into which people would insert their smartphone in front of their eyes. They would access group therapy using the online application, vTime (vTime, Limited, H., 2018). During group therapy, the therapists and the patients would be anonymous and represented by an avatar (an abstract character).

\section{Procedure}

To accommodate the two different participant groups (patients and therapists), two separate topic guides were developed. The patient topic guide was developed with an independent (local) service user and career involvement group (Simpson et al., 2013), and the therapist topic guide was developed with two therapists with experience delivering group therapy. The questions and the prompts in the two topic guides were comparable with each other and intended to discover what participants thought of a groupbased CBT intervention delivered using VR.

MD conducted all interviews in private locations that were convenient to the participants. These locations included a research office, therapists' offices, and patients' homes. At the beginning of the interviews, participants were asked to provide written informed consent and fill in a brief demographic questionnaire. The information provided on the questionnaires was used to observe the diversity of the participants and to ensure a spread of different professional backgrounds and experience.

The interviews began with the researcher (MD) reading the description of the proposed intervention from the topic guide (see above). Participants then watched a 38-second demonstration video of the proposed intervention (https:/youtu.be/ OjZYXF6w6g4). Following this, participants trialed the VR technology using a smart-phone and HMD. The VR application tested was V-time (www.vtime.net). This is a social networking service where people can connect to other people in a virtual space. Each person is represented in the virtual space by an 
avatar. During the first 15-20 min of the hour-long interview, participants trialed the technology. They were verbally guided by the interviewer through the $\mathrm{V}$-time application and encouraged to test different features such as customizing their avatars and testing at least three virtual environments of their choice. Unfortunately, due to time constraints and ethical reasons, participants could not interact with another avatar online. However, when trialing the technology and watching the demonstration video, they knew what talking to an avatar would entail. At the end of the interviews, patients received $£ 15$ reimbursement for their participation.

\section{Data Analysis}

Demographic data collected from participants were imported into IBM SPSS statistics version 24 software, and descriptive analysis was performed. Audio recordings from the interviews were transcribed verbatim by the two authors conducting the data analysis. This was done to develop familiarization with the data (Riessman, 1993). The first author (MD) transcribed 75\% of the recordings, and the second author (LH) transcribed $25 \%$. Transcripts were imported into QSR NVivo version 12 qualitative data analysis software.

Thematic analysis was used to analyze the qualitative data (Braun and Clarke, 2006). The authors approached the data analysis with a pragmatic viewpoint, whereby themes identified had to have practical implications for current group therapy practice (Agerfalk, 2010). The data were analyzed inductively for the first research question and deductively for the second research question. The NICE guidelines for the delivery of GCBT for the management and treatment of depression were used as the deductive framework (NICE, 2018). The analytic process was recursive, with the authors moving back and forth throughout the six phases. The phases included familiarization, generating initial codes, searching for themes, reviewing themes, defining the themes, and writing the report (Braun and Clarke, 2006).

All transcripts were coded line by line by the transcribing author, and through extensive discussion, a framework of thematic codes was created. Individual extracts of data were then sorted into codes. A semantic (descriptive) level of analysis was conducted, where all codes were read, and patterns within the data that were relevant to the research questions were conceptualized as preliminary themes. The author (MD) presented these preliminary themes to a peerreview meeting of research psychologists $(n=23)$ that has extensive experiences in researching social interactions in mental health care, including developing and evaluating innovative interventions (Queen Mary University of London, 2020). The established research group meets once a week to provide constructive feedback to developing research projects. The hour-long meeting was used to discuss potential broader meanings and implications of the preliminary themes. Feedback from this process was then used to revise the themes. The emerging overarching themes and subthemes were reviewed using Patton (2002) dual criteria, where themes had to have internal homogeneity and external heterogeneity. Finally, the findings and the narrative of the themes and subthemes were discussed and agreed on with the remaining authors (DA, SP, and VB).

\section{RESULTS}

\section{Participants}

Ten patients with a current diagnosis of depression participated. Half of the patients were female (50\%), and most of them identified as White British (70\%). On average (mean), the patients were 49 years of age (SD 12.23) and had a diagnosis of depression for an average of 16.7 years (SD 12.60). More than half of the patients $(60 \%)$ had experience attending verbal group therapy in the community. All were aware of VR (100\%), but only a minority (20\%) had used it before this study, for gaming (50\%) and other purposes (50\%).

Ten CBT therapists with varying levels of experience participated. Half of the therapists were female (50\%) and most identified as White British (80\%). On average (mean), therapists had been working in mental health for 14.2 years (SD 7.84) and had been facilitating group-based verbal therapy for 7.1 years (SD 3.90). All therapists were aware of VR (100\%), and the majority (70\%) had used it before the study. Therapists used virtual reality for gaming and leisure purposes (42.9\%) and professionally to deliver treatment $(42.9 \%)$ and receive training (14.3\%). A summary of participant demographics can be found in Table 2.

\section{Themes}

Five themes were identified in relation to the two research questions. An overview of themes and the related subthemes can be found in Table 3. Themes were mapped onto the research questions. Verbatim participant quotes have been provided alongside the analysis. Additional quotes supporting the results can be found. Throughout the results, themes discussed by both participant groups will be reported as "participants," whereas those discussed by a specific participant group will be reported with the participant group name (e.g., patients or therapists).

\section{WHAT ARE THE VIEWS OF POTENTIAL STAKEHOLDERS ON CBGT DELIVERED VIA VR?}

\section{Accessibility to Treatment}

Participants highlighted some facilitators and barriers to accessing VRGT.

\section{Facilitators of Treatment}

The convenience of receiving treatment remotely from home was widely referenced as an advantage of VRGT. The majority of participants felt that this feature of VRGT would improve access to evidence-based treatment. The patient population that was expected to benefit the most from this feature was patients who struggle to leave the house due to physical disabilities (e.g., bedbound or wheelchair-using patients). VRGT would give 
TABLE 2 | Demographic characteristics of the sample.

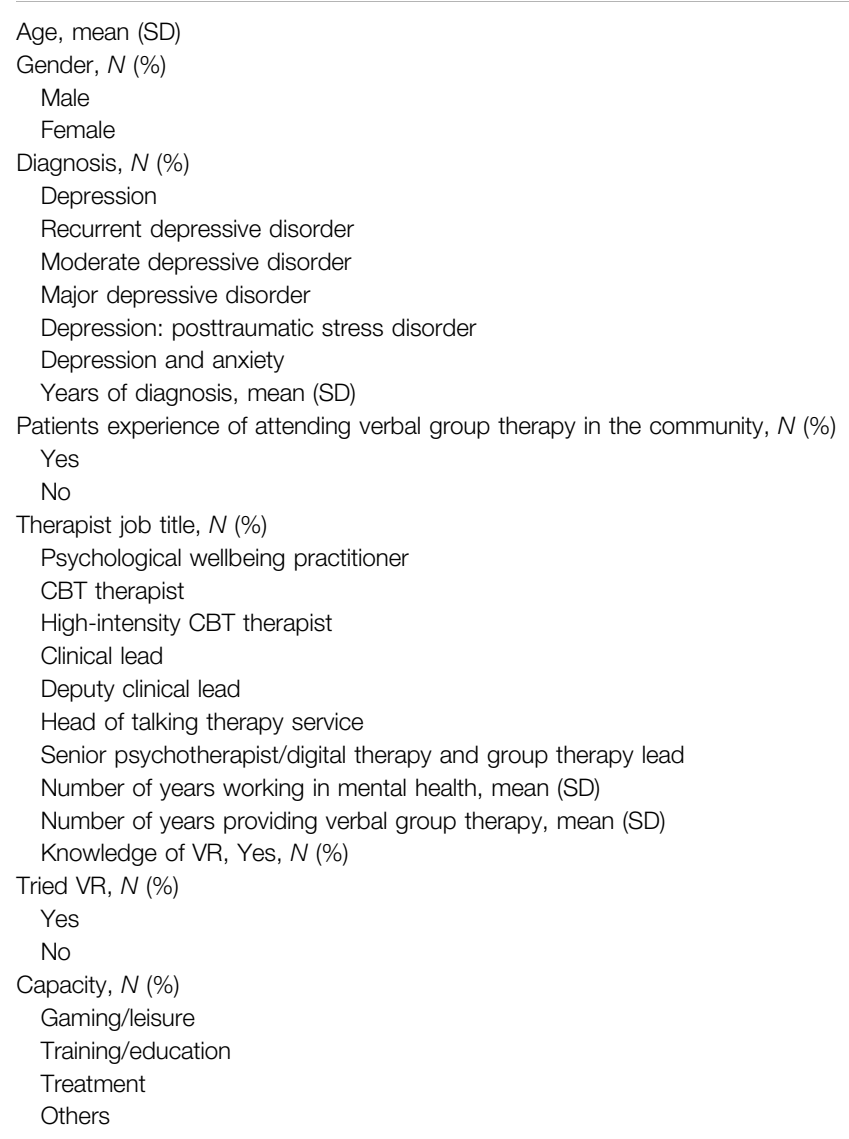

Patients $(\mathrm{N}=10)$

Pationts $(N=10)$

Therapists $(\mathbf{N}=10)$

\begin{tabular}{|c|c|}
\hline 49 (12.23) & 39 (10.61) \\
\hline 5 (50\%) & 5 (50\%) \\
\hline $5(50 \%)$ & 5 (50\%) \\
\hline $4(40 \%)$ & $\mathrm{N} / \mathrm{A}$ \\
\hline \multicolumn{2}{|l|}{2 (20\%) } \\
\hline \multicolumn{2}{|l|}{1 (10\%) } \\
\hline \multicolumn{2}{|l|}{1 (10\%) } \\
\hline \multicolumn{2}{|l|}{$1(10 \%)$} \\
\hline \multicolumn{2}{|l|}{1 (10\%) } \\
\hline $16.70(12.60)$ & N/A \\
\hline $6(60 \%)$ & $\mathrm{N} / \mathrm{A}$ \\
\hline \multicolumn{2}{|l|}{$4(40 \%)$} \\
\hline \multirow[t]{7}{*}{$\mathrm{N} / \mathrm{A}$} & 2 (20\%) \\
\hline & 2 (20\%) \\
\hline & 2 (20\%) \\
\hline & 1 (10\%) \\
\hline & 1 (10\%) \\
\hline & 1 (10\%) \\
\hline & 1 (10\%) \\
\hline $\mathrm{N} / \mathrm{A}$ & $14.22(7.84)$ \\
\hline N/A & 7.10 (3.90) \\
\hline 10 (100\%) & 10 (100\%) \\
\hline $2(20 \%)$ & 7 (70\%) \\
\hline $8(80 \%)$ & 3 (30\%) \\
\hline 1 (50\%) & 3 (42.9\%) \\
\hline- & 1 (14.3\%) \\
\hline- & 3 (42.9\%) \\
\hline 1 (50\%) & - \\
\hline
\end{tabular}

\section{TABLE 3 | Themes and subthemes.}

\begin{tabular}{|c|c|c|c|c|c|}
\hline \multirow{2}{*}{$\begin{array}{l}\text { Research } \\
\text { questions } \\
\text { Themes }\end{array}$} & \multicolumn{3}{|c|}{$\begin{array}{l}\text { What are the views of potential stakeholders on } \\
\text { CBGT delivered via VR? }\end{array}$} & \multicolumn{2}{|c|}{$\begin{array}{l}\text { How can CBGT intervention be adapted } \\
\text { to be delivered via VR? }\end{array}$} \\
\hline & $\begin{array}{l}\text { 1. Accessibility to } \\
\text { treatment }\end{array}$ & $\begin{array}{l}\text { 2. Patient engagement during } \\
\text { treatment }\end{array}$ & $\begin{array}{l}\text { 3. Future of mental } \\
\text { health }\end{array}$ & $\begin{array}{l}\text { 4. Recommendations to available } \\
\text { software }\end{array}$ & 5. Treatment structure \\
\hline \multirow[t]{3}{*}{ Subthemes } & $\begin{array}{l}\text { 1.1. Facilitators of } \\
\text { treatment }\end{array}$ & 2.1. The use of avatars & & 4.1. Patient assessments & $\begin{array}{l}\text { 5.1. Adjustments to treatment } \\
\text { guidelines }\end{array}$ \\
\hline & $\begin{array}{l}1.2 \text { Barriers of } \\
\text { treatment }\end{array}$ & $\begin{array}{l}\text { 2.2. Immersive virtual } \\
\text { environments }\end{array}$ & & 4.2. One-to-one meetings & $\begin{array}{l}\text { 5.2. Blended vs pure VR } \\
\text { treatment }\end{array}$ \\
\hline & & & & $\begin{array}{l}\text { 4.3. Worksheets and whiteboards } \\
\text { 4.4. Homework }\end{array}$ & \\
\hline
\end{tabular}

this subgroup of patients a sense of achievement as they would feel like they have been somewhere and feel less socially isolated as they are remotely connected to other patients. The second subgroup of patients that were expected to benefit from the remote nature of VRGT are patients with depression and anxiety who lack the motivation to leave their house or are anxious to go to group therapy. Thirdly, patients who have to work during service hours can access the group treatment remotely from their workplace, preferably during their lunch break. Finally, as the remoteness of VRGT removes geographical barriers, patients living in remote locations would be able to have access to group therapy.

Not having to sort of make an effort to go to a location, cause sometimes even that can be quite, just the thought of you know I have got to make my way there, that can be quite difficult sometimes if you are not in the right headspace. (P1, Patient) 
In addition to the remote nature of VRGT, a few participants who were partially sighted or had hearing loss found that the headsets could be easily adapted to their needs, e.g., moving the screen closer or increasing the volume. The participants highlighted that these features might also encourage people to access the treatment.

You see for me; I could probably see this group better than the group in real life because it is just right here, so I would not be able to see people sitting that far away from me. It is clear when it is right on your screen. . I can see better with this [HMD] on. (P18, Therapist)

Despite recognition that the remoteness of VR can enable access to treatment without leaving the house, a few participants questioned whether this type of therapy was promoting isolation and avoidance for depressed patients who already lacked the motivation to leave the house.

If someone is depressed and they spend all their time at home in virtual reality and [on a] computer and you do this [VRGT] on top of that they could be potentially feeling or getting into avoidance and stuff maybe. (P19, Therapist)

\section{Barriers of Treatment}

Participants highlighted some barriers that might prevent them from accessing VRGT. The barriers discussed could be split into stakeholder and technological barriers.

\section{Stakeholder Barriers}

Participants were clear that VRGT might not be for everybody (P2, patient). People's perception of this type of treatment was one of the most cited barriers. Some participants already viewed group therapy as an inferior treatment to one-to-one therapy, and the possibility of delivering or receiving this substandard treatment remotely through VR frustrated them. If patients were offered VRGT, some wanted to know why they were offered this over conventional therapies.

I think at first when you say VR. . that kind of almost feels like a double fob-off because you are like, well not only are you sending me to this group therapy but you are not even sending me to group therapy (laughs) like doing whatever this is. (P3, Patient)

Technical ability was discussed as a potential stakeholder barrier to VRGT. As the average age of the patients interviewed was 49 years old, some patients were concerned that they might not have the technical skills to access the group treatment virtually. They recommended that this treatment is aimed at younger patients who are more "techsavvy." On the other hand, some patients feared missing out and did not want there to be an age limit on this treatment. They suggested that it should be offered to everyone regardless of their age and that patients should have the choice to decide whether they would like to try VRGT.
Younger people I guess are more tech-minded, and they are into everything so they would know, but if you are an older person, it might feel a little daunting, it might feel alien. (P1, Patient)

In contrast to some patients' views regarding age and technological ability, therapists have highlighted that technology has been around long enough for older patients to develop the technical skills required for this type of treatment. Furthermore, they highlighted that the elderly patients were more adherent with the remote technology-based treatments that are currently on offer.

We do SilverCloud [online CBT] here, which is a digital platform, and a lot of the people that do really well on Silver Cloud are people aged 50+, so that is a digital intervention. So I think we probably live in an age now where digital has been around for quite a while, so it is probably not so much of a stigma for people. (P20, Therapist)

To access VRGT, participants would require a smartphone and a wireless network connection (Wi-Fi). A few participants would not have access to a smartphone because they had mobile phones suited to their physical disabilities, e.g., amplified mobile phones for hearing loss or phones with large buttons for partially sighted users. Furthermore, some participants may not be able to afford a monthly Wi-Fi connection or a smartphone. The cost of the equipment has raised the issue as to whether a person's financial situation might be a barrier to accessing VRGT. Some participants did not want to be disadvantaged because of money and recommended that they come into services where they can access free $\mathrm{Wi}-\mathrm{Fi}$ or that there is a loan program for smartphones. Therapists argued that one of the unique selling points of VRGT is that it is accessed remotely. If participants travel to a service for this type of treatment, they should be offered conventional faceto-face group therapy. Furthermore, therapists were not keen on loaning equipment as they were worried about how it would be looked after and whether they would get the equipment back.

I do not have the right kind of phone. I have a phone that
is more suitable for my particular kind of disabilities, and
my main disability is... my hearing and my vision. . and
some people might not be able to run a phone at all. If
they are living on basic benefits, they might not be able to
run a mobile [phone]. (P2, Patient)

Finally, participants who had previously used VR recreationally highlighted that some people might experience motion sickness or eye strain with VR and that this might act as a barrier to group therapy.

When I have used virtual reality before just a little bit here and there recreationally same sort of headset, you do sometimes find that you get a bit motion sick looking around... [However,] it is hard to know cause I think if you are just sitting still and looking around a little bit, it 
is probably ok, it is the sort of simulations where you are going on a roller coaster and looking around, there is something weird, but it's still something worth checking out. (P19, Therapist)

\section{Technical Barriers}

Upon testing the VR technology, a few participants reported technical issues that might interrupt the running of a smooth group treatment or prevent people from accessing their treatment. Three technical barriers were reported: (1) Participants smartphones not being fully charged to cover the duration of the treatment; (2) lateness to the treatment as a result of the set-up and loading time needed for the hardware (HMD) and software (V-time); and (3) potential background noise coming through participants microphones interrupting members' concentration. Participants recommended that they should receive some training on the technology and that some of these potential issues are covered before the treatment in the ground rules, e.g., participants should access this treatment in a quiet room of the house. Furthermore, participants requested that there is technical support available if any technical issues occur during the treatment.

57, 65, 69, 70, 79 loading, so you probably need to start about 10 minutes before to get ready, would we? (P2, Patient)

\section{Patient Engagement during Treatment}

The majority of the participants underlined how group therapy delivered using avatars could improve therapeutic engagement. Avatars increase the anonymity of patients and limit the nonverbal cues received from other group members. For instance, a patient talking about their problems cannot see whether other patients are judging them or look bored and disengaged. Therefore, the use of avatars was widely referenced as a factor that might encourage sharing and consequently contribute to the improvement of therapeutic engagement. The use of immersive virtual environments was another factor that was thought to potentially increase therapeutic engagement.

\section{The Use of Avatars}

Most patients openly spoke about their anxieties with face-to-face group therapies and felt that the use of avatars in VRGT would reduce some of these anxieties. A common anxiety expressed by patients was whether another patient in the group would identify them. The underlying concerns here were whether someone they knew would hear their anxieties and whether they were going to feel stigmatized for attending mental health treatment. The anonymity provided by avatars was thought to reduce the perceived stigma in attending group treatment. Patients may no longer need to be worried about being identified by other group members, and this could potentially improve patient's attendance to group treatment. The potential of avatars in reducing patients' anxieties of being identified was also echoed by therapists.
There is definitely some worry about 'what if I know somebody there' that sort of thing, but if you are looking at an avatar or person distanced from you a little bit, in some way, it might make some likely to engage, more likely to turn up, likely to give it a go as well. (P11, Therapist)

Another concern patients experienced was regarding their physical appearance. Patients highlighted that, as a result of their depression, they were not always motivated to take care of their physical appearance. Therefore, when attending face-toface group therapy, they felt self-conscious and were concerned about what other people might think of them. Furthermore, patients who had visible physical disabilities (e.g., wheelchair users) reported that, during group therapy, their physical disability generally overshadows the mental health issues they want to discuss. Patients were excited that their avatars could potentially look like how they wanted them to. This could potentially give them the confidence to attend group treatment even if they do not feel presentable. Furthermore, for patients with physical disabilities, it potentially allows them to be present in the group with just their mental health issues.

My [wheel] chair would not have to be obvious ... I
would be sitting on an ordinary chair in that room with
those people... In that kind of therapy [VRGT] there is
no difference to anybody else apart from the fact that we
have all got a mental health disorder of some description,
so I am no different to anybody else. . Having an avatar
like that would make me equal to everybody else so it
would start to make me heal. Straight up it would make
me on the same level on the same footing as everybody
else apart from our mental health disorders. (P2, Patient)

In contrast to patients who felt empowered, a couple of therapists reported feeling disempowered by the use of avatars. Therapists were worried that if patients selected avatars that were not a close representation of themselves, therapists might be treating a person rather than the actual patient. Furthermore, they argued that having avatars that were not an accurate representation of the patient might prevent forming of trusting relationships in the group. To offset these potential issues, therapists recommended setting boundaries regarding how different avatars can be from the patient or as an icebreaker in the first session allowing patients to report whether their avatars look similar to them.

In terms of people forming trusting relationships. . my concern would be, how would that work, I mean it is still possible, but it would be interesting to see, do people feel connected to other people, I think maybe what is going to be important to people is that person being authentically them as they can be, like I was saying like if I have dreadlocks but turn up in blond hair like that is not me being actually me. I [would] like to see an avatar that looks like someone personally. (P16, Therapist) 
The final concern patients shared was other patients' and therapists' opinions of them. Patients who have attended face-toface group therapy before recognized that, due to fears of being physically attacked, they often hold back their honest opinions about other patients. As a consequence of this, they are aware that other patients may also not be honest with their opinions, and this leads to them overanalyzing other members' nonverbal cues. While disclosing an issue, a nonverbal cue that made patients feel judged was other group members looking at each for validation of their response.

When I'm there [face-to-face group therapy] sometimes I want to talk but ... if I was to do this one [VRGT], I think I would be more confident to talk to say exactly how I am feeling, without seeing other peoples... how they are looking at me, you know if they are judging me, sometimes I don't want to say things in case I'm being judged. (P9, Patient)

Avatars reduce nonverbal cues in a virtual environment, and participants felt that this feature, combined with the anonymity provided by avatars, would inevitably increase disclosures in group therapy. Furthermore, therapists discussed how more open and honest conversations within the group could create cohesion and potentially improve group dynamics.

You may lose out on some of the subtle non-verbal cues, one of the benefits you may find is that it may help create cohesion in a group cause we often find clients often rub each other the wrong way because of non-verbal cues. (P13, Therapist)

Despite the majority of participants implying that the use of avatars would improve therapeutic engagement, a few patients associated the use of avatars with gaming and found the use of avatars as disrespectful to therapy and mental health. On the other hand, other patients acknowledged that use of avatars would be fun at the beginning of a session and potentially act as an icebreaker between patients, but then, as patients start discussing mental health issues, there will be a shift into a serious atmosphere.

Cartoon characters [avatars] some people might actually think that was fun, but to me, it is a little bit of a mockery towards mental health, I think it is you know the fact that you have cartoon characters, but at the same time you are talking about something really serious that has affected that person, I don't know, I do not like the idea of that,... it reminds me too much of video games and playing whereas therapy is serious and you have to take it serious. . . so I do look at it a little bit as a mockery to therapy and to mental health. (P6, Patient)

\section{Immersive Virtual Environments}

During VRGT, the therapist can conduct the sessions in a variety of different immersive virtual environments, e.g., on a beach, under the northern lights, or by a wilderness river. At the beginning of the interviews, participants got the opportunity to try some of these immersive virtual environments and comment on them. All the participants were pleasantly surprised at how realistic and immersive these virtual environments looked. Patients very quickly highlighted that these virtual environments allowed them to visit locations that they could not afford or were not physically able to visit. Therapists found the virtual environments therapeutically valuable and discussed how they could use them in their sessions (e.g., guided imagery).

\section{Incredible oh my god how amazing cause you can just imagine... [Although] you know that it is not real [but] your [brain] quickly catches up, doesn't it, with the fact that it is real, it feels like it is real, that you could be part of that environment, how amazing. (P20, Therapist).}

The majority of the participants found the virtual environments calming and distracting simultaneously. Some participants felt that this combination would relax patients enough to want to talk about their issues and therefore engage in the group treatment. In contrast, other participants felt that there might be too many distractions to focus on what is being discussed.

I lose confidence very quickly in a group where they are judging me... having something to focus on while I am talking, something that grabs my attention, like the aurora borealis and stuff like that keeps me relaxed enough to want to talk. (P2, Patient)

Despite finding most virtual locations calming and relaxing, a few participants acknowledged that specific virtual environments (e.g., shipwreck) might not be appropriate or therapeutic. Furthermore, other virtual environments that seem therapeutic (e.g., a beach) might unknowingly trigger an upsetting memory for some patients (e.g., if a patient has had a bad experience with water). It was implied that therapists should start the therapy in a neutral virtual environment (e.g. boardroom) and then through discussion and negotiation allow the group to select locations they find appropriate.

Maybe there's some locations that might make some people feel more at ease could make some other people feel more anxious. (P10, Patient)

Although the majority of the participants agreed that virtual environments could be therapeutically valuable, a few participants were concerned about patients' physical environments (e.g., home) during VRGT. Female participants highlighted that some single parents might not have access to child-free spaces at home where they can fully immerse themselves in a virtual environment.

Some people are single parents to one or more children and sitting and not being able to keep their eyes on their children or not being able to have somebody there to take 
care of their children whilst they sit for half an hour in a different location and not be disturbed by their children would be [a barrier]. (P2, Patient)

Therapists were intrigued at how accessing group treatment through virtual environments might impact the patient's treatment. Some therapists argued that physically distancing patients away from their everyday environments and conducting therapy in a clinical environment allowed patients to step out of their everyday situations and reflect on their life.

\section{A lot of what we, how therapy works, what we touch on is the idea that having, coming into an environment, coming into an office where your physically distanced from your situation can have its benefits. So, coming into a new clinical environment as such allows you to take a step out of the situation and allows you to see it in a different light. So, I would be curious as to whether or not that will still occur in virtual reality. (P10, Therapist)}

However, contrary to this argument, a therapist spoke about the feedback he received from a patient receiving remote therapy. The patient had argued that conducting therapy in her environment made therapy more relevant to her and prevented her from compartmentalizing her problem and therapy. Furthermore, a patient who discussed the issue of physical locations implied that they preferred using virtual environments because it distributed the power between the therapist and the patients and made them feel more in control of their treatment. This may improve therapeutic engagement in treatment.

It is very unnerving to come to any kind of therapists office because, as a service user you lose all power and control really when you are in someone else's office or someone else's building. (P3, Patient)

\section{Future of VR Technology in Mental Health}

This theme was predominantly discussed by therapists. Therapists not only viewed VRGT as an acceptable treatment method but they also discussed how VR technology could potentially revolutionize services in the future. As the treatments would be delivered remotely, therapists felt that VRGT would be cost-saving to IAPT services as they would no longer need large buildings with so many rooms to deliver group therapy.

It is the way of the future, and I am very excited. I think it would be great for a service like ours, we have done so much, but we no longer have the capacity in this building to hold people, and so this would allow us to hold groups regularly without needing the space, so I think that would be a major benefit. (P12, Therapist)

Currently, IAPT services are split into boroughs, and patients are offered treatments that are available in their local IAPT service. As a result of the remote nature of VR, therapists highlighted that, in the future, patients may no longer be limited to services offered in their borough, but could also access specialized services delivered in different boroughs. This could potentially lead to distributing specialist resources across the country and a reduction of waitlist times as patients could receive treatments from less busy services. Patients accessing specialized treatment quicker might lead to a reduction in the revolving door phenomenon, where patients repeatedly present at services. However, for some therapists, patients potentially receiving treatments from different boroughs have raised questions regarding how the NHS would distribute funds to local IAPT services. To overcome issues of funding, a few therapists imagined a central technological intervention delivery center. This center would have therapists that have training in delivering technology-based therapeutic interventions like VRGT and would receive patient referrals from across the country.

It can be challenging in terms of if you get referrals from other areas then how does that work in terms of money and funding. (P16, Therapist)

Contrary to the views of therapists, a few patients that discussed VR technology and the future of mental health did so negatively. Patients highlighted that they were not comfortable with the speed of technological development and felt that if they did not keep up with these developments, they were going to be left out.

I wish we could slow it down. . technology is [going to] control every aspect of life shortly, I mean I have tried to pull away, I only have an email address now, nothing else, but you would be punished for this in the end. (P7, Patient)

\section{HOW CAN CGBT INTERVENTION BE ADAPTED TO BE DELIVERED VIA VR?}

The second research question was to address how the CGBT intervention could be adapted to be delivered via VR. The NICE guidelines for the delivery of CGBT for the management and treatment of depression were used as the deductive coding framework.

\section{Recommendations to Available Software}

CBT aims to teach patients how to identify and change unhelpful thinking, "it is part of learning theory" (P18, therapist), which is why delivering this type of therapy often requires therapists to rely on patient assessments, worksheets, a whiteboard and homework. Therefore, the practicalities of running CBGT using VR might be challenging. The social networking application trialed for this study (V-time) was not developed for mental health treatment purposes. It, therefore, did not have some of the inbuilt features that would make delivering CBGT convenient for therapists. To overcome the limitations of using a free and inadequate application, and to stay true to the nature of CBT, therapists made some recommendations. 


\section{Patient Assessments}

Before each group, CBT session patients are generally asked to fill in a clinical assessment form. Therapists score and use these forms to track their patient's development and to identify who might be at risk to themselves or others. Some IAPT services use paper assessment forms, whereas other services have moved onto an electronic system, where patients use a weblink to fill in the questions online, and the results are sent directly to their patient file and therapist. Depending on whether or not their service had access to an electronic platform influenced whether or not therapists were worried about how they would collect patient assessments before a group. Therapists working in services that used paper assessments recommended patients' email or verbally disclose their scores during treatment. However, this suggestion has raised some confidentiality concerns and was quickly discarded.

We email it [assessments] to them, they go to a link, fill in the questionnaire, submit and then it goes into their clinical contact for that day. So it is already there before [the session], and so you know how they have been feeling that week. It is a good thing that we have introduced. (P17, Therapist)

\section{One-To-One Meetings}

In group therapy, it is common for patients and therapists to have a quick one-to-one catch up. These catch ups commonly occur before a session, during a coffee break or at the end of the session. Patients value this as it gives them the space to discuss issues they are not comfortable discussing in a group environment. Therapists like to use these openings to talk to patients who have scored high on assessments and are either a potential risk to themselves or others. It may not be feasible to conduct one-to-one catch ups using V-time. Therapists saw an advantage to this limitation that, by removing social contact with therapists, VR might encourage patients to keep discussions within the group. Therapists were curious as to how this change might impact group cohesion. To manage patients presenting with potential risk, therapists recommended giving these patients a call at the end of the session. This 'courtesy call' would mimic the individual conversations which therapists have with their patients. To make sure this recommendation was cost-effective, most therapists agreed that this call should only be for patients who are at risk to themselves or others.

With the telephone calls, yes, we absolutely have a duty of care to manage risk and if people kind of indicate distress or need for a follow-up we do that. Having said that, we know that one of the things that needs to be done right in groups is that everything needs to be addressed in the groups so I would be quite wary about addressing things outside the group because then group cohesion is affected and all the other group processes. (P15, Therapist)

\section{Worksheets and Whiteboard}

CBT therapists often use worksheets and whiteboards to illustrate cognitive processes to their patients. The possibility of conducting a group CBT session without worksheets or a whiteboard concerned junior therapists more than senior therapists. Therapists who had experience delivering one-to-one CBT though other online applications, such as WebEx, ideally wanted the VR application to have a document share feature. The V-time application has a photo share feature, in which therapists can share photos of the worksheets or illustrations with group members. However, not being fully aware of this feature, an idea that was explored to aid therapists was a patient treatment file. This paper file would be sent to patients' homes, and in the order of the sessions that will be conducted, it will contain all the worksheets, illustrations and homework's patients would beed in the session.

So we have a treatment manual so there would be a patient manual that they receive. I suppose it would be needed to be adapted for these sessions, so they would have a description of the content and the worksheets and everything else that they might need, so that's there. (P15, Therapist)

\section{Homework}

Homework is an essential feature of CBT treatment. Currently, therapists set patients paper-based homework (e.g., mood diaries) and check they have been completed in follow-up sessions. Checking paper-based homework might be difficult in a virtual environment. Therefore, therapists have recommended setting practical rather than paper-based homework that patients can verbally feedback. A few therapists even recommended using other VR applications for homework. These recommendations were welcomed by patients that found paper-based homework "pointless" (P3, patient) and reported that they often did not complete them. Patients wanted to be encouraged to complete real-life tasks that they can relate to.

I think you probably could do some homework with VR; you might be able to do things like...I guess engaging with other people via VR, you know... friends whatever. Definitely exposure is quite a good one, so when you want people to get exposed to a particular thing, ... .bringing things to reality as possible, I think that is quite a good one. I think some people might find it quite exciting to do virtual reality, sort of, homework. (P14, Therapist)

\section{Treatment Structure}

NICE guidelines have evidence-based recommendations for the delivery of group CBT. They recommend group sessions to consist of nine sessions for $90 \mathrm{~min}$ and have two facilitators supporting up to twelve patients per group. These recommendations are for face-to-face group CBT. Therefore, during the interviews, participants were asked how appropriate these recommendations are for VR delivery. 


\section{Adjustments to Treatment Guidelines}

IAPT services offer slight variations (eight to ten sessions) to the recommended nine CBGT sessions. Therefore, participants found the recommended nine sessions reasonable for VRGT. Aside from the number of sessions, the majority of the participants recommended adjustments to be made to the NICE guidelines for VRGT. A majority of the participants highlighted that 90 min was too long to wear a headset and be immersed in a virtual environment. When therapists conduct 90 min sessions, they usually include a short break for patients to smoke or use the bathroom. If VRGT was 90 min long, therapists were concerned that they might struggle to bring patients back from a break. On the other hand, patients reported that, due to their depression, they struggle to concentrate for long durations. Therefore, they preferred shorter treatment sessions.

If everyone is in the same room, then you can say 'ok break,' and then you can gather people back together. I do not know how you would do that in VR, that might be a bit trickier so potentially reducing the amount of time and making it a more concise group session rather than full ninety minutes. (P12, Therapist)

Despite the majority of the participants favoring shorter group sessions, a couple of participants felt that 90 -min is a perfect length of time (P1, Patient) as it allowed patients to get comfortable in a group and discuss their issues.

Although NICE recommends that CBT groups should be facilitated by two therapists, the majority of the therapists found this humorous and reported that, due to the limited number of therapists they have, two therapists per group does not happen in reality. Therefore, therapists recommended having one CBT-trained therapist to facilitate the group.

So the recommendations are lovely recommendations, and in an ideal world that would be a perfect way to deliver [CBT therapy] but the reality is very different, resource-wise we do not have [two therapists per group]. The reason why I rolled my eyes was because we have so many groups and were trying to offer so many groups to fit with the recommendations, but unfortunately, we do not have the staff to be able to facilitate or co-facilitate. So we very often have one facilitator. (P20, Therapist)

Groups with large numbers of people may not be practical for VRGT. Patients reported feeling anxious in large groups and preferred having fewer patients in groups as it gave them a chance to engage and discuss what they wanted. A therapist who had experience delivering group CBT through WebEx reported the difficulty in managing large groups in technological environments. As nonverbal cues are lost in these types of groups, if group sizes are large and they are not managed appropriately, it frequently leads to people talking over each other. Similarly, other therapists recommended keeping the groups small so that they can remember whose avatar is whose.
In the real world it is a bit easier to manage a big group, somehow it would be easier to get a sense of who they are, whereas if you got that [VR] the visual cues are not as strong, so it might be hard to keep track of voices and knowing who is who. (P19, Therapist)

\section{Pure vs. Blended VR Treatment}

As part of the treatment structure, participants discussed whether VRGT should be offered with no (pure) or some face-to-face contact (blended treatment). Patients' preferences varied widely; some felt scared to meet other patients face-to-face and argued that the treatment should be kept purely to VR. However, others, who use group therapies to build relationships, wanted to be given a choice at the end of the VR therapy to meet up in person. Contrarily, a few patients did not want to meet other patients but initially meet the therapist and see what they are all about (P10, Patient).

I don't really think it's necessary to mix face to face contact with the VR. I think especially if you're going to do the face to face contact at the beginning, that's terrifying. If you're going to do face-to-face contact at the end, then I personally would have that in the back of my mind that like, 'I'm going to meet these people in real life and they're going to be real'. And that would kind of ramp up the anxiety. (P3, Patient)

In contrast to patients, therapists consistently recommended keeping VRGT pure with no face-to-face contact. Therapists claimed that the unique selling point of VRGT is that it is convenient and anonymous for patients, and blending the treatment with face-to-face contact would counteract these advantageous features. Furthermore, therapists argued that, by keeping the mode of treatment consistent throughout, they could better manage patients' expectations and avoid patients feeling like they are missing out on treatment with face-to-face contact. Finally, therapists who have experience delivering group therapy using technology also highlighted that consistency with the mode of treatment gives better treatment outcomes.

It should be virtual reality all the way through. . . otherwise, you will miss some of the selling points, some of the people may like the fact that they are anonymous. I do online typed therapy and a lot of people like not meeting their therapist, that is a massive selling point. . they don't get caught up on the content base of their thought because they are not concerned about some of the judgements from the therapist. (P13, Therapist)

\section{DISCUSSION}

\section{Summary of Key Findings}

Our findings indicate that many stakeholders not only viewed VRGT as an acceptable treatment method but also as a method that might modernize treatment delivery in the future. Several factors that positively influenced VRGT's acceptability were identified. These factors included (1) the convenience of 
receiving treatment remotely from home, encouraging patients to access evidence-based treatment and (2) during VRGT, the anonymity and reduction of nonverbal cues provided by avatars and the immersive virtual environments improving therapeutic engagement and group cohesion.

Stakeholders also identified factors that might prevent some patients from accessing VRGT and therefore affecting its acceptability. These barriers included patients' view that this form of treatment might be less effective than face-to-face treatment and their technical abilities and concerns about cost (to afford smartphones and Wi-Fi). Additionally, stakeholders raised some technical barriers, e.g., smartphone battery duration, software loading time, and potential background noise during treatment, that may prevent patients and therapists from finding VRGT accessible.

To deliver group CBT using VR, certain adjustments need to be made to the conventional treatment structure, e.g., making sessions shorter and reducing the number of patients per group. Furthermore, to compensate for the lack of adequate VR treatment software, therapists need to adjust their way of working. These adjustments include using electronic patient assessments, courtesy calls for individual discussions, patient treatment file, or online document share for treatment worksheets and practical homework.

\section{Strengths and Limitations}

To our knowledge, this is the first study that has explored the acceptability of a proposed VRGT intervention for people with depression. Conducting this study using a qualitative method has enabled valuable insight into what factors the potential intervention users (therapists and patients with depression) think may affect the acceptability and uptake of this intervention (Yardley et al., 2015).

This study was conducted in the United Kingdom and was imbedded in NHS IAPT services. The pragmatic approach adopted has meant that the findings have practical recommendations for this context.

Despite these strengths, the study has some limitations. Firstly, the interviews were conducted before the coronavirus pandemic, when group videoconferencing was not the norm. Now that people have adapted to connecting with family and friends virtually, their perceptions of VRGT might have changed. However, exposure to technology and virtual group meetings has likely had a positive impact on the acceptability of this type of treatment.

Whilst testing the VR technology during interviews, for ethical and logistical reasons, participants were not able to interact with other participants avatars. Instead, they tested the technology and watched a demonstration video of what VRGT would look like. The lack of avatar interaction may have had an impact on the feedback which participants gave regarding the use of avatars. However, from the feedback received, it can be observed that participants had a good idea of how avatars would work.

\section{Comparison with the Literature}

Consistent with the digitally enabled health literature, this study has found that participants perceive that the proposed VRGT intervention might reduce the time and travel associated with treatment, the perceived stigma, and potentially the cost of services (Price et al., 2014; Fairburn and Patel, 2017; Bucci et al., 2019; Ralston et al., 2019). In reducing these potential barriers, it aims to enhance access to evidence-based care and more actively engage patients in their treatment (Price et al., 2014).

Findings from this study also align with the concerns highlighted in previous literature regarding the use of digital health technologies. These include patients and therapists' perceptions of this kind of treatment being inferior and a cheap replacement of face-to-face contact and this potentially impacting the uptake and implementation of the treatment and the potential for technological systems to fail (Bucci et al., 2019).

This study has found that the anonymity provided by avatars and the minimization of authority provided by virtual environments could lead to increased disclosure and better group cohesion. This finding supports the "online disinhibition effect," which is a term used to describe the phenomena of people feeling more comfortable to express themselves in cyberspace than in real life (Suler 2004). When people use cyberspace positively for acts of kindness or selfdisclosure, it is called benign disinhibition, and this could be considered as a tool to understand the self and to resolve interpersonal problems. In contrast, online disinhibition could be toxic, where people use cyberspace to make harsh comments to each other. This could be a space for catharsis (the release of strong, honest feelings).

Cohesiveness, self-understanding, and catharsis are therapeutic factors involved in the development of group therapy (Yalom and Crouch, 1990). Therefore, findings from this study indicate that VR may be a valuable therapeutic space to conduct group therapy. Contrary to this, some therapeutic factors needed for an effective group environment might not be present in VR, for instance, imitative behavior is a therapeutic factor, and the lack of nonverbal cues from the use of avatars may be a barrier to people imitating each other. Similarly, most face-to-face groups offer an opportunity for peer support and development of socializing techniques. However, these therapeutic factors may not be available in a virtual group that is delivered remotely.

\section{Implications}

The findings from this study have immediate clinical implications. To comply with social distancing measures during the coronavirus pandemic, mental health services have swiftly started to use online videoconferencing technology (e.g., Zoom and WebEx) to deliver group therapy remotely to their patients. The speed at which social distancing measures were put into place has meant that mental health services are using trial and error methods to explore the acceptability of remote online group treatments. As discussed previously, the pragmatic approach adopted in this study has meant that the findings yield themselves to practical recommendations. Therefore, therapists could use the recommendations in this study to deliver their virtual group treatments.

This study has found VRGT to be an acceptable treatment method for many patients with physical disabilities. Furthermore, it has identified features that make VRGT accessible (e.g., remoteness, adaptability of technology to physical needs, and 
concealment of physical disability). Researchers could use knowledge developed from this study to develop technological interventions suited more for this patient population, and therapists could use the findings to be aware of these patients needs and offer them more suitable treatments.

In addition to clinical and research implications, findings from this study could be used by software developers to develop an application specifically for the delivery of group CBT. The application could have all the necessary CBT features (e.g., whiteboard function) and would, therefore, limit the barriers faced by a free application.

\section{CONCLUSION}

Although the findings suggested that VRGT may be more acceptable for some patients than for others, overall, the response of the patients and therapists was largely positive. The treatment may improve accessibility, therapeutic engagement and potentially transform how mental health treatment is delivered in the future. However, for CBT to be implemented into VRGT, certain adjustments need to be made to the software used and the conventional face-to-face treatment delivery.

\section{DATA AVAILABILITY STATEMENT}

The original contributions presented in the study are included in the article/Supplementary Materials, and further inquiries can be directed to the corresponding author.

\section{REFERENCES}

Agerfalk, P. J. (2010). Getting pragmatic. Eur. J. Inf. Syst. 19 (3), 251-256. doi:10. 1057/ejis.2010.22

Arthur, S., Mitchell, M., Lewis, J., and Nicholls, C. (2014). “Designing fieldwork,” In Qualitative research practice a guide for social science students and researchers. 2nd Edn (London, United Kingdom: SAGE), 147-176

Braun, V., and Clarke, V. (2006). Using thematic analysis in psychology. Qual. Res. Psychol. 3, 77-101. doi:10.1191/1478088706qp063oa

Braun, V., and Clarke, V. (2013). Successful qualitative research: a practical guide for beginners. 1st Edn (United Kingdom: SAGE). doi:10.1002/jmr.2361

Bucci, S., Schwannauer, M., and Berry, N. (2019). The digital revolution and its impact on mental health care. Psychol Psychother. 92 (2), 277-297. doi:10.1111/ papt.12222

Carr, C. E., O'Kelly, J., Sandford, S., and Priebe, S. (2017). Feasibility and acceptability of group music therapy vs wait-list control for treatment of patients with long-term depression (the SYNCHRONY trial): study protocol for a randomised controlled trial. Trials. 18 (1), 1-15. doi:10.1186/s13063-0171893-8

Clark, D. M. (2011). Implementing NICE guidelines for the psychological treatment of depression and anxiety disorders: the IAPT experience. Int. Rev. Psychiatr. 23 (4), 318-327. doi:10.3109/09540261.2011.606803

Cuijpers, P., Van Straten, A., and Warmerdam, L. (2008). Are individual and group treatments equally effective in the treatment of depression in adults? A metaanalysis. Eur. J. Psychiatr. 22 (1), 38-51. doi:10.4321/S021361632008000100005

Dilgul, M., Martinez, J., Laxhman, N., Priebe, S., and Bird, V. (2020). Cognitive behavioural therapy in virtual reality treatments across mental health

\section{ETHICS STATEMENT}

The studies involving human participants were reviewed and approved by East Midlands-Leicester South committee (ref: 19/ EM/0217). The patients/participants provided their written informed consent to participate in this study.

\section{AUTHOR CONTRIBUTIONS}

MD designed, conducted, analyzed, and wrote the study. LH analyzed the results. DA was responsible for recruitment of participants. SP and VB designed the study and supervised and edited the article.

\section{FUNDING}

This study has been funded by the East London NHS Foundation Trust as part of a PhD project. The funding of the East London Foundation Trust supported this research, and the researchers at the Unit for Social and Community Psychiatry provided feedback on an early draft of this paper.

\section{ACKNOWLEDGMENTS}

The authors wish to acknowledge Newham talking therapies, Catherine Carr and Jasmine Martinez, for assisting with the recruitment for this study.

conditions: a systematic review. Consortium Psychiatricum. 1 (1), 30-46. doi:10.17650/2712-7672-2020-1-1-30-46

Dilgul, M., Mcnamee, P., Orfanos, S., Carr, C. E., and Priebe, S. (2018). Why do psychiatric patients attend or not attend treatment groups in the community: a qualitative study. PLoS One. 13(12), 1-16. doi:10.1371/journal.pone.0208448

Dobson, C. (2008). Conducting research with people not having the capacity to consent to participation: a practical guide for researchers. United Kingdom: British Psychological Society

Fairburn, C. G., and Patel, V. (2017). The impact of digital technology on psychological treatments and their dissemination. Behav. Res. Ther. 88, 19-25. doi:10.1016/j.brat.2016.08.012

Fenn, K., and Byrne, M. (2013). The key principles of cognitive behavioural therapy. InnovAiT: Education and Inspiration for General Practice. 6 (9), 579-585. doi:10.1177/1755738012471029

Fernandez, E., Salem, D., Swift, J. K., and Ramtahal, N. (2015). Meta-analysis of dropout from cognitive behavioral therapy: magnitude, timing, and moderators. J. Consult. Clin. Psychol. 83 (6), 1108-1122. doi:10.1037/ ccp0000044

Freeman, D., Reeve, S., Robinson, A., Ehlers, A., Clark, D., Spanlang, B., et al. (2017). Virtual reality in the assessment, understanding, and treatment of mental health disorders. Psychol. Med. 47(14), 2393-2400. doi:10.1017/ S003329171700040X

Hans, E., and Hiller, W. (2013). Effectiveness of and dropout from outpatient cognitive behavioral therapy for adult unipolar depression: a meta-analysis of nonrandomized effectiveness studies. J. Consult. Clin. Psychol. 81 (1), 75-88. doi:10.1037/a0031080

Huntley, A. L., Araya, R., and Salisbury, C. (2012). Group psychological therapies for depression in the community: systematic review and meta-analysis. $\mathrm{Br}$. J. Psychiatry. 200 (3), 184-190. doi:10.1192/bjp.bp.111.092049 
Jerald, J. (2015). “What is virtual reality?," In The VR book: human-centered design for virtual reality. 1st Edn, Editor J. Jerald (London, United Kingdom: ACM Books), 9-12

Kösters, M., Burlingame, G. M., Nachtigall, C., and Strauss, B. (2006). A metaanalytic review of the effectiveness of inpatient group psychotherapy. Group Dynam. 10 (2), 146. doi:10.1037/1089-2699.10.2.146

Knowles, L. M., Stelzer, E. M., Jovel, K. S., and O'Connor, M. F. (2017). A pilot study of virtual support for grief: feasibility, acceptability, and preliminary outcomes. Comput. Hum. Behav. 73, 650-658. doi:10.1016/j.chb.2017.04.005

Maples-Keller, J. L., Bunnell, B. E., Kim, S.-J., and Rothbaum, B. O. (2017). The use of virtual reality technology in the treatment of anxiety and other psychiatric disorders. Harv. Rev. Psychiatr. 25 (3), 103-113. doi:10.1097/HRP. 0000000000000138

McDermut, W., Miller, I. W., and Brown, R. A. (2001). The efficacy of group psychotherapy for depression: a meta-analysis and review of the empirical research. Clin. Psychol. Sci. Pract. 8 (1), 98-116. doi:10.1093/clipsy.8.1.98

NHS Mental Health Taskforce. (2016). The five year forward view for mental health. England: Independent Mental Health Taskforce, 1-81.

NHS. (2018). Psychological therapies, annual report on the use of IAPT services. England: NHS digital, 1-2.

NICE. (2018). Depression in adults: recognition and management. Retrieved fromhttps://www.nice.org.uk/guidance/cg90/resources/depression-in-adultsrecognition-and-management-pdf-975742638037

Norcross, J. C., Pfund, R. A., and Prochaska, J. O. (2013). Psychotherapy in 2022: a delphi poll on its future. Prof. Psychol. Res. Pract. 44 (5), 363. doi:10.1037/ a0034633

Nosek, M. A., Robinson-whelen, S., Hughes, R. B., and Nosek, T. M. (2016). An Internet-based virtual reality intervention for enhancing self-esteem in women with disabilities: results of a feasibility study. Rehabil. Psychol. 61 (4), 358-370. doi:10.1037/rep0000107

O’Brien, B. C., Harris, I. B., Beckman, T. J., Reed, D. A., and Cook, D. A. (2014). Standards for reporting qualitative research. Acad. Med. 89 (9), 1245-1251. doi:10.1097/acm.0000000000000388

Patton, M. Q. (2002). Qualitative research and evaluation methods. 3rd Edn. Thousand Oaks, CA: Sage.

Price, M., Yuen, E. K., Goetter, E. M., Herbert, J. D., Forman, E. M., Acierno, R., et al. (2014). mHealth: a mechanism to deliver more accessible, more effective mental health care. Clin. Psychol. Psychother. 21 (5), 427-436. doi:10.1002/cpp.1855

Queen Mary University of London. (2020). Unit for social and community Psychiatry. Retrieved from https://www.qmul.ac.uk/socialpsych/ (October 19, 2020)

Ralston, A. L., Andrews, A. R., and Hope, D. A. (2019). Fulfilling the promise of mental health technology to reduce public health disparities: review and research agenda. Clin. Psychol. Sci. Pract. 26 (1), 1-14. doi:10.1111/cpsp.12277

Riessman, C. K. (1993). Narrative analysis. Qualitative research methods. Sage. United Kingdom: SAGE.

Sampaio, M., Haro, M. V., De Sousa, B., Melo, W. V., and Hoffman, H. (2020). Therapists make the switch to telepsychology to safely and continue treating their patients during the covid-19 pandemic. virtual reality telepsychology may be next. Front. Virtual Real. doi:10.3389/frvir.2020.576421

Sekhon, M., Cartwright, M., and Francis, J. J. (2017). Acceptability of healthcare interventions: an overview of reviews and development of a theoretical framework. BMC Health Serv. Res. 17 (1), 1-13. doi:10.1186/s12913-0172031-8

Simpson, A., Jones, J., Barlow, S., and Cox, L. (2013). Adding SUGAR: service user and carer collaboration in mental health nursing research. J. Psychosoc. Nurs. Ment. Health Serv. 52 (1), 22-30. doi:10.3928/02793695-20131126-04

Suler, J. (2004). The online disinhibition effect. Cyberpsychol. Behav. 7 (3), 321-326. doi:10.1089/1094931041291295

Tucker, M., and Oei, T. P. S. (2007). Is group more cost effective than individual cognitive behaviour therapy? The evidence is not solid yet. Behav. Cognit. Psychother. 35 (1), 77. doi:10.1017/S1352465806003134

vTime, Limited, H. (2018). vTime-the sociable network. Retrieved fromhttps:// vtime.net/ (November 25, 2020)

Watson, A. J., Grant, R. W., Bello, H., and Hoch, D. B. (2008). Brave new worlds: how virtual environments can augment traditional care in the management of diabetes. J Diabetes Sci Technol. 2 (4), 697-702. doi:10.1177/ 193229680800200422

Williams, M. (2016). "Pragmatism," In Key concepts in the philosophy of social research. 1st Edn, Editor C. Rojek (London, United Kingdom: SAGE), 170-175

Wykes, T., Parr, A. M., and Landau, S. (1999). Group treatment of auditory hallucinations: exploratory study of effectiveness. Br. J. Psychiatry. 175 (2), 180-185. doi:10.1192/bjp.175.2.180

Yalom, I. D., and Crouch, E. C. (1990). The theory and practice of group psychotherapy. Br. J. Psychiatry. 157 (2), 304-306. doi:10.1192/ s0007125000062723

Yardley, L., Morrison, L., Bradbury, K., and Muller, I. (2015). The person-based approach to intervention development: application to digital health-related behavior change interventions. J. Med. Internet Res. 17 (1), 30. doi:10.2196/jmir. 4055

Yeo, A., Legard, R., Keegan, J., Ward, K., Nicholls, C., and Lewis, J. (2014). In-depth interviews. In Qualitative research practice a guide for social science students and researchers. 2nd Edn Editors J. Ritchie, J. Lewis, C. Nicholls, and R. Ormston (London, United Kingdom: SAGE), 177-210

Conflict of Interest: The authors declare that the research was conducted in the absence of any commercial or financial relationships that could be construed as a potential conflict of interest.

Copyright $(2021$ Dilgul, Hickling, Antonie, Priebe and Bird. This is an open-access article distributed under the terms of the Creative Commons Attribution License (CC $B Y)$. The use, distribution or reproduction in other forums is permitted, provided the original author(s) and the copyright owner(s) are credited and that the original publication in this journal is cited, in accordance with accepted academic practice. No use, distribution or reproduction is permitted which does not comply with these terms. 